\title{
Novel SINS Alignment Method Using SG-KP-QKF
}

\author{
Changyan Ran \\ College of Computer and Information Technology, Three Gorges University, Yichang 443002, P. R. \\ China
}

Email: ranchangyan@126.com

Keywords: SINS, alignment, SG-KP-QKF.

Abstract. This paper proposes a novel direct and completely nonlinear alignment method for the strapdown inertial navigation system (SINS). The method uses the sparse-grid quadrature Kalman filter based on the Kronrod-Patterson rule (SG-KP-QKF) and nonlinear models. The system equations and the measurement equations are all nonlinear. One of the advantages of the method is that the nonlinear SG-KP-QKF directly output the Euler angles which are simultaneously updated in the process of filter time updating. Finally, the method is verified by inertial measurement unit (IMU) experimental data. The alignment results show that the proposed method can obtain an accurate initial attitude within a short convergence time. These findings suggest that the proposed method differs from traditional alignment methods and can thus be considered a easier solution for SINS alignment.

\section{Introduction}

The alignment process is very important for a strapdown inertial navigation system (SINS). The dead-reckoning algorithm will use the initial orientation to update the attitude information.

The alignment condition is usually rough and is full of all kinds of noise and disturbance, to the extent that the initial coarse attitude errors are large. Moreover, the SINS system models are nonlinear, thus nonlinear filters are more proper than Kalman filter. The extended Kalman filter, the unscented Kalman filter[1], the quadrature Kalman filter[2], the cubature Kalman filter[3,4], the sparse-grid quadrature Kalman filter[5,6], and the sparse-grid quadrature Kalman filter based on the Kronrod-Patterson rule[7] among others are proposed. Compared with the EKF and UKF, the estimation accuracy level of SG-KP-QKF can be flexibly controlled. In addition, the SG-KP-QKF is more efficient than the QKF with similar performance[7].

A novel alignment method is proposed. When SINS does the alignment, the body linear velocity is usually zero. Under this condition, the method uses the SG-KP-QKF as the nonlinear filter and compares the local gravity vector with the specific force. The advantage of this approach is that the outputs of the nonlinear SG-KP-QKF are directly the Euler angles, the updating of which is simultaneously realized in the filter time updating. Thus, navigation computation is no longer needed. Finally, the method is verified by IMU experimental data, and the alignment results show that the method can obtain accurate initial attitude. The method is very simple and easy to implement, as well as provides a new solution for SINS alignment.

The remainder of this paper is organized as follows: Section II presents the SINS alignment models, including the system equations and the measurement equations. Section III discusses the SG-KP-QKF algorithm. Section IV demonstrates the alignment performance of the novel method by using IMU experimental data on a three-axis turntable. Section V concludes.

\section{SINS Alignment Models}

The propagation of the Euler angles, is governed by the following differential equations:

$$
\left[\begin{array}{l}
\theta \propto \\
\gamma \\
\psi \propto
\end{array}\right]=\frac{1}{\cos \theta}\left[\begin{array}{ccc}
\cos \theta \cos \gamma & 0 & \sin \gamma \cos \theta \\
\sin \theta \sin \gamma & \cos \theta & -\cos \gamma \sin \theta \\
-\sin \gamma & 0 & \cos \gamma
\end{array}\right] \omega_{n b}^{b}
$$

where $\theta, \gamma, \psi$ are the pitch, roll and heading Euler angles. 
Assuming that the vehicle linear velocity is zero, $\boldsymbol{\omega}_{n b}^{b}$ can be simplified as

$$
\boldsymbol{\omega}_{n b}^{b}=\boldsymbol{\omega}_{i b}^{b}-\boldsymbol{C}_{n}^{b} \boldsymbol{\omega}_{i e}^{n}
$$

where $\omega_{i b}^{b}=\left[\omega_{i b x}^{b}-\delta \omega_{i b x}^{b}, \omega_{i b y}^{b}-\delta \omega_{i b y}^{b}, \omega_{i b z}^{b}-\delta \omega_{i b z}^{b}\right]^{T}, \omega_{i b x, y, z}^{b}$ and $\delta \omega_{i b x, y, z}^{b}$ are the gyroscope outputs and errors along $x$, $y, z$-axis of body.

The fiber gyroscope errors and the accelerometer errors are modeled as the sum of a Gaussian white noise and a bias expressed in the $b$-frame, which are defined as

$$
\begin{aligned}
& \delta \omega_{i b x}^{b}=\varepsilon_{g x}+w_{g x}, \delta \omega_{i b y}^{b}=\varepsilon_{g y}+w_{g y}, \delta \omega_{i b z}^{b}=\varepsilon_{g z}+w_{g z} \\
& \delta f_{i b x}^{b}=\nabla_{a x}+w_{a x}, \delta f_{i b y}^{b}=\nabla_{a y}+w_{a y}, \delta f_{i b z}^{b}=\nabla_{a z}+w_{a z}
\end{aligned}
$$

The instrument bias dynamics may be represented by

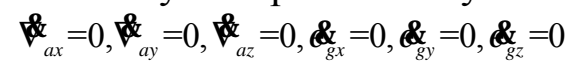

The state vector can be expressed in component form as

$$
\boldsymbol{x}=\left[\theta, \gamma, \psi, \nabla_{a x}, \nabla_{a y}, \nabla_{a z}, \varepsilon_{g x}, \varepsilon_{g y}, \varepsilon_{g z}\right]^{T}
$$

The system noise vector can be written as

$$
\boldsymbol{w}=\left[w_{g x}, w_{g y}, w_{g z}, 0,0,0,0,0,0\right]^{T}
$$

where $\boldsymbol{w}$ is normally distributed (Gaussian) with a power spectral density of $\boldsymbol{Q}$.

According to the selected state vector and the system noise vector, Eqs. 1 and 5 can be combined and expressed in state space form as

$$
\boldsymbol{x}=\boldsymbol{F}(\boldsymbol{x})+\boldsymbol{G}(\boldsymbol{x}) \boldsymbol{w}
$$

where $\boldsymbol{F}(\boldsymbol{x})$ is the nonlinear state transfer function, and $\boldsymbol{G}(\boldsymbol{x})$ is the system noise input function.

A fourth-order Runge-Kutta scheme with two steps between successive inertial sensor sampling instances is employed for numerical integration. Then the continuous system equation is converted to a difference equation to give

$$
\boldsymbol{x}_{k}=\boldsymbol{F}\left(\boldsymbol{x}_{k-1}\right)+\boldsymbol{w}_{k-1}
$$

where $\mathrm{E}\left[\boldsymbol{w}_{k}\right]=0, \mathrm{E}\left[\boldsymbol{w}_{k} \boldsymbol{w}_{j}^{T}\right]=\boldsymbol{Q}_{k} \boldsymbol{\delta}_{k j}, \boldsymbol{Q}_{k}=\boldsymbol{G}\left(\boldsymbol{x}_{k}\right) \boldsymbol{Q} \boldsymbol{G}\left(\boldsymbol{x}_{k}\right)^{T} T_{1}, \boldsymbol{G}\left(\boldsymbol{x}_{k}\right)$ is the discrete form of $\boldsymbol{G}(\boldsymbol{x})$ at time $t_{k}$, and $T_{1}$ is the discrete time interval.

Assuming that the vehicle linear velocity is zero, for a terrestrial navigation system operating in the local geographic reference frame, the navigation equation can be simplified as

$$
\boldsymbol{g}^{n}=-\boldsymbol{f}^{n}
$$

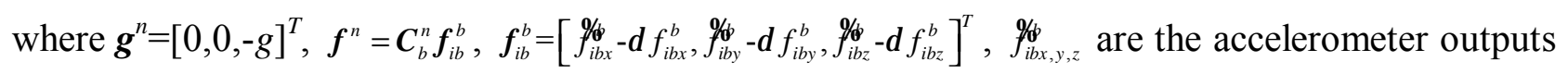
along $x, y, z$-axis of body.

$\boldsymbol{g}^{n}$ is a constant vector that we can set as the reference measurement. Hence, measurement vector can be expressed as

$$
z=[0,0,-g]^{T}
$$

The measurement noise vector can be expressed as

$$
\boldsymbol{\eta}=\left[w_{a x}, w_{a y}, w_{a z}\right]^{T}
$$

where $\eta$ is modeled as additive Gaussian white noise with a power spectral density of $\boldsymbol{R}$.

According to Eq.11, Eq.12, and Eq.6, the measurement equation (Eq.10)is can be written in state-space form as

$$
z=\boldsymbol{H}(\boldsymbol{x})+\boldsymbol{U}(\boldsymbol{x}) \boldsymbol{\eta}
$$

where $\boldsymbol{H}(\boldsymbol{x})$ is the nonlinear measurement function, and $\boldsymbol{U}(\boldsymbol{x})$ is the measurement noise input function.

The measurement at time $t_{k}$ can be expressed in terms of the state as

$$
\boldsymbol{z}_{k}=\boldsymbol{H}\left(\boldsymbol{x}_{k}\right)+\boldsymbol{\eta}_{k}
$$

where $\mathrm{E}\left[\boldsymbol{\eta}_{k}\right]=0, \mathrm{E}\left[\boldsymbol{\eta}_{k} \boldsymbol{\eta}_{j}^{T}\right]=\boldsymbol{R}_{k} \boldsymbol{\delta}_{k j}, \boldsymbol{R}_{k}=\boldsymbol{U}\left(\boldsymbol{x}_{k}\right) \boldsymbol{R} \boldsymbol{U}\left(\boldsymbol{x}_{k}\right)^{T} / T_{2}, T_{2}$ is the discrete time interval, $\mathrm{E}\left[\boldsymbol{w}_{k} \boldsymbol{\eta}_{j}^{T}\right]=0$. We assume that $T_{2}$ and $\mathrm{T}_{1}$ are the same as the sensor data updating cycle $T$. 


\section{SG-KP-QKF Algorithm}

The SG-KP-QKF utilizes the Kronrod-Patterson rule to determine the univariate quadrature point sets with a range of accuracy levels, which are then extended for the multi-dimensional point sets using the sparse-grid technique. Xianghong et.[7] give the detailed process to generate the univariate quadrature point sets and the multi-dimensional point sets of the SG-KP-QKF. According to the reference[7], we can calculate the multi-dimensional points and weights of the SG-KP-QKF as shown in Table 1.

Table 1 Quadrature point sets of the SG-KP-QKF $(L=3, d=9)$

$$
\left\{\begin{array}{l}
a_{j}=\left\{\begin{array}{l}
2, j=1 \\
-0.2778, j=2, \mathrm{~L}, 19 \\
0.0278, j=20, \mathrm{~L}, 163
\end{array}\right. \\
\xi_{j}=\left\{\begin{array}{l}
{[0,0, \mathrm{~L}, 0]^{T}, j=1} \\
{[0, \mathrm{~L}, \pm \sqrt{3}, \mathrm{~L}, 0]^{T}, j=11, \mathrm{~L}, 19} \\
{[0, \mathrm{~L}, \pm \sqrt{3}, \mathrm{~L}, \pm \sqrt{3}, \mathrm{~L}, 0]^{T}, j=20, \mathrm{~L}, 163}
\end{array}\right.
\end{array}\right.
$$

If the univariate point sets are determined based on the Gauss-Hermite rule[5], and such filter is named as the SG-GH-QKF and to minimize the total number of multi-dimensional point sets, the multi-dimensional points and weights are shown in Table 2.

Table 2 Quadrature point sets of the SG-GH-QKF $(L=3, d=9)$

$$
\left\{\begin{array}{c}
a_{j}=\left\{\begin{array}{c}
34, j=1 \\
-4, j=2, \mathrm{~L}, 19 \\
0.1667, j=20, \mathrm{~L}, 37 \\
0.25, j=38, \mathrm{~L}, 181
\end{array}\right. \\
\xi_{j}=\left\{\begin{array}{c}
{[0,0, \mathrm{~L}, 0]^{T}, j=1} \\
{[0, \mathrm{~L}, \pm 1, \mathrm{~L}, 0]^{T}, j=2, \mathrm{~L}, 19} \\
{[0, \mathrm{~L}, \pm 1.732, \mathrm{~L}, 0]^{T}, j=20, \mathrm{~L}, 37} \\
{[0, \mathrm{~L}, \pm 1, \mathrm{~L}, \pm 1, \mathrm{~L}, 0]^{T}, j=38, \mathrm{~L}, 181}
\end{array}\right.
\end{array}\right.
$$

where $\left(\xi_{j}, a_{j}\right)$ are the quadrature points and corresponding weights.

From Tables 1 and 2, under the condition of the same level $L$ and dimension $d$, the total quadrature point number of the SG-KP-QKF is 163 , and the total quadrature point number of the SG-GH-QKF is 181. Thus, the nest feature of the SG-KP-QKF univariate points is more efficient than that of SG-GH-QKF.

\section{Simulation}

The simulation data are collected from a prototype navigation grade IMU on a three-axis turntable. The turntable rotates along its three vertical axes, the swaying frequency is $0.2 \mathrm{~Hz}$. The swaying amplitude is $5^{\circ}$. To varify the accuracy of the initial alignment, near the end of the alignment, the turntable stops at the position of which the heading is $0^{\circ}$. The sensor data are sampled at $200 \mathrm{~Hz}$. The simulation total time is 30 minutes, of which the first 5 minutes are used for the coarse alignment. The last 25 minutes are used for the fine alignment. The three accelerometer biases and noises are all less than $0.2 \mathrm{mg}$, whereas the three gyroscope biases and noises are all less than $0.03 \circ / h$. The initial latitude is $26.5017^{\circ} \mathrm{N}$, and longitude is $106.71667^{\circ} \mathrm{E}$.

The attitude errors at the end of the fine alignment are shown in Table 3.

Table 3 Attitude errors

\begin{tabular}{lll}
\hline Pitch error & Roll error & Heading error \\
$-0.54^{\prime}$ & $0.28^{\prime}$ & $11.62^{\prime}$ \\
\hline
\end{tabular}


The pitch and roll errors converge rapidly in approximately 20 seconds. The heading alignment error converges more slowly than tilt errors, and the convergence time is approximately 60 seconds. This alignment feature is similar to that of the traditional methods.

Fig. 1 shows the figures of the Euler angles in the last 2 minutes of fine alignment.

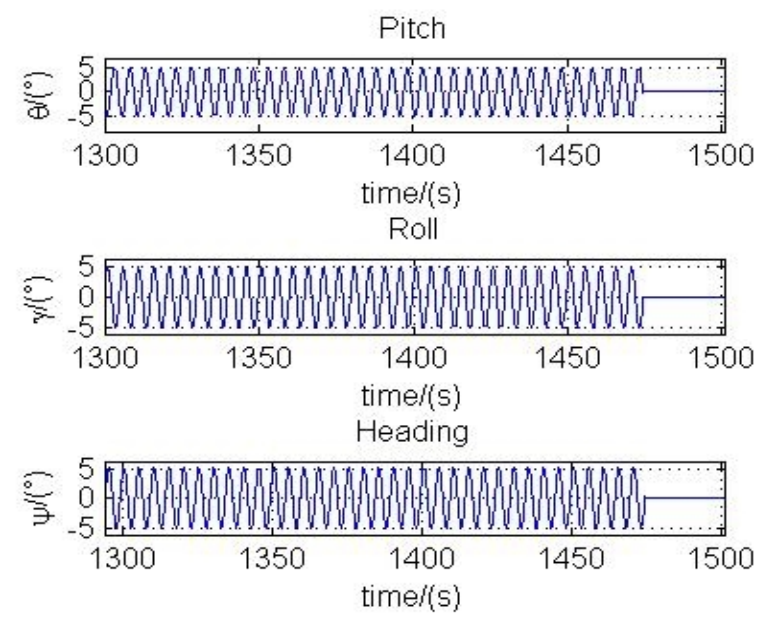

Fig. 1 Euler angles

According to the above discussion and simulation results, both the alignment errors and convergence time can meet the requirements of alignment. Thus, the novel direct and nonlinear SINS alignment method using the SG-KP-QKF is proven practical.

\section{Summary}

In this paper, a novel direct and nonlinear SINS alignment method using the SG-KP-QKF is proposed. The results of the simulation using IMU experimental data show that the novel alignment method can achieve small alignment errors in a short convergence time and can thus effectively accomplish the initial alignment. Furthermore, compared with the conventional SINS alignment methods, the proposed method is easier to apply. The system and measurement equations are primarily the navigation equations without any approximations, which make the models very simple. The results of this paper prove that the proposed method is a novel idea for SINS alignment.

\section{References}

[1] S. J. Julier, J. K. Uhlmann, and H. F. Durrant-Whyte, A new method for the nonlinear transformation of means and covariances in filters and estimators, IEEE Trans. Autom. Control. 45(2000) 477-482.

[2] K. Ito and K. Xiong, Gaussian filters for nonlinear filtering problems, IEEE Trans. Autom. Control. 45(2000) 910-927.

[3] I. Arasaratnam and S.Haykin, Cubature kalman filters, IEEE Trans. Autom. Control. 54(2009) 1254-1269.

[4] I. Arasaratnam and S. Haykin, Cubature Kalman smoothers, Automatica, 47(2011) 2245-2250.

[5] B.Jia, M.Xin and Y.Cheng, Sparse Gauss-Hermite quadrature filter with application to spacecraft attitude estimation, J. Guid. Control, Dynam. 34(2011) 367-379.

[6] B.Jia, M.Xin and Y.Cheng, Sparse-grid quadrature nonlinear filtering, Automatica, 48(2012)327-341. 
[7] C. Xianghong, R.Changyan and W. Haipeng, Sparse-grid Quadrature Kalman Filter based on the Kronrod-Patterson rule, 2013 I2MTC, Minneapolis, MN. (2013) 1396-1401. 